PAPER

\title{
Parkinsonism following bilateral lesions of the globus pallidus: performance on a variety of motor tasks shows similarities with Parkinson's disease
}

\author{
M Kuoppamäki, J C Rothwell, R G Brown, N Quinn, K P Bhatia, M Jahanshahi
}

J Neurol Neurosurg Psychiatry 2005;76:482-490. doi: 10.1136/jnnp.2003.020800

See end of article for authors' affiliations

Correspondence to:

$\operatorname{Dr} M$ Jahanshahi, Sobell

Department of Motor

Neuroscience and

Movement Disorders,

Institute of Neurology,

Queen Square, London

WCIN 3BG, UK

m.jahanshahi@

ion.ucl.ac.uk

Received 15 June 2003

In revised form

2 August 2004

Accepted 7 August 2004
Objectives: The authors report the results of detailed investigations into the motor function of a patient who, after a heavy drinking binge and subsequent unconsciousness, respiratory acidosis, and initial recovery, developed parkinsonism characterised by hypophonic speech and palilalia, "fast micrographia", impaired postural reflexes, and brady/akinesia in proximal (but not distal) alternating upper limb movements.

Methods: In addition to brain magnetic resonance imaging (MRI), different aspects of motor function were investigated using reaction time (RT) tasks, pegboard and finger tapping tasks, flex and squeeze tasks, movement related cortical potentials (MRCPs), and contingent negative variation (CNV). Cognitive function was also assessed. The results were compared to those previously reported in patients with Parkinson's disease (PD).

Results: Brain MRI showed isolated and bilateral globus pallidus (GP) lesions covering mainly the external parts $(\mathrm{GPe})$. These lesions were most probably secondary to respiratory acidosis, as other investigations failed to reveal an alternative cause. The results of the RT tasks showed that the patient had difficulties in preparing and maintaining preparation for a forthcoming movement. MRCP and CNV studies were in line with this, as the early component of the MRCP and CNV were absent prior to movement. The patient's performance on pegboard and finger tapping, and flex and squeeze tasks was normal when performed with one hand, but clearly deteriorated when using both hands simultaneously or sequentially.

Conclusions: In general, the present results were similar to those reported previously in patients with PD. This provides further indirect evidence that the output of globus pallidus is of major importance in abnormal motor function in PD. The possible similarities of the functional status of GP in PD and our case are discussed.
T here is considerable interest in the pathophysiology of motor dysfunction in different basal ganglia diseases, especially in idiopathic Parkinson's disease. Preparation and execution of voluntary movements assessed using different reaction time paradigms, ${ }^{1-3}$ or through recording of movement related cortical potentials (MRCP) $)^{4-6}$ have been extensively studied in PD. The overall conclusion is that the preparation, initiation, and the execution of voluntary movements are slow or abnormal in patients compared with healthy control subjects. ${ }^{7}$ It is also known that bradykinesia in PD becomes more prominent by repetitive, simultaneous, or sequential movements. ${ }^{7}$ Despite these interesting results, it has been very difficult to examine the role of individual nuclei in the pathophysiology of motor dysfunction in PD, as the underlying alterations in different pathways and nuclei are complex in this disease. ${ }^{8}$ Although the gross morphology of the globus pallidus (GP) is normal, ${ }^{10}$ it is widely accepted that the abnormal functional output of GP is one of the major contributors to motor dysfunction in PD. ${ }^{89}$ The renaissance in the surgical treatment of PD has provided a new tool with which to investigate basal ganglia function. In addition to pallidal surgery, isolated pallidal lesions offer another opportunity to study the significance of this nucleus in different motor functions. However, such lesions are rare, ${ }^{11}$ and we found only 12 reported cases of isolated bilateral pallidal lesions from the literature. To date, there is only one case report of bilateral lesions of the globus pallidus (GP), with an additional lesion of the right substantia nigra, in which numerous electrophysiological tests were performed to study different aspects of motor function. ${ }^{12}$ The results showed delayed and slow movements of the upper limbs during different reaction time paradigms and while performing a fast goal directed arm movement task. ${ }^{12}$

We now describe a case with acquired isolated bilateral lesions of the GP, on whom a variety of behavioural and electrophysiological measures were obtained to investigate different aspects of motor function. These aspects of motor function in our case, and the role of GP, are discussed by comparing the present results to previously published results of healthy control subjects and patients with PD.

\section{CASE DESCRIPTION}

Our patient was a civil servant working with electronics. He had a past history of alcohol but no disulfiram, methanol, or narcotic drug abuse. He was admitted to hospital at the age of 26 years in a disorientated state after a severe drinking binge. Shortly afterwards, he lapsed into an unconscious state and had several generalised seizures. Investigations at that stage indicated a severe respiratory acidosis. However, he recovered quickly and was discharged from the hospital.

About three months after this episode he noted problems with his writing which had become small and untidy. Over the next three years these symptoms gradually progressed.

Abbreviations: CNV, contingent negative variation; CRT, choice reaction time; GP, globus pallidus; IOL, inter-onset latency; MRCPs, movement related cortical potentials; NART, National Adult Reading Test; PD, Parkinson's disease; RMF, Recognition Memory for faces; RMW, Recognition Memory for Words; RT, reaction time; SRT, simple reaction time 
On examination, three years later, he had a mildly abnormal gait with dystonic external rotation of the left foot. He could walk heel to toe normally and hop on either leg, but his arm swing was slightly reduced on the right. His speech was hypophonic and he manifested palilalia, and festination and freezing of speech. His face was hypomimic and he had blepharoclonus. Pursuit and voluntary saccadic eye movements were normal. The glabellar tap was positive but there were no other primitive reflexes and the jaw jerk was absent. Rapid tongue movements were normal. Voluntary palatal movements and the gag reflex were normal. Postural reflexes were impaired. Although his axial tone was increased, there was no limb rigidity. He had no tremor, and the speed and amplitude of repeated finger and hand movements and tapping tasks were normal with no fatigue. However, alternate supination and pronation of the arms revealed a dramatic deficit with rapid breakdown of rhythm, timing, and amplitude and fatigue over 5-10 repetitions. On writing there was "fast micrographia", ${ }^{13}$ so that although the size of his writing was extremely small, its speed was normal. He could, however, use his hands normally for doing up buttons, tying laces, and pressing computer keys. Tapping tasks with his feet were difficult, as they were interrupted by freezing. Muscle power in the limbs and deep tendon reflexes were normal, and the plantar responses were flexor. He had no abulia. Treatment with trihexiphenidyl and levodopa had no effect. His family history was negative.

Routine blood counts, blood smears for acanthocytes, serum biochemistry including liver function tests, copper and caeruloplasmin, autoantibody screen, syphilis serology, alfa-fetoprotein, lactate, and pyruvate levels, white cell enzymes, and plasma amino acids, were all normal or negative. DNA analysis was negative for mitochondrial mutations (8344 and 8993). EEG, EMG, and nerve conduction studies and visual evoked potentials were normal. Brain MRI revealed bilateral and symmetrical signal changes (hypointensity in $\mathrm{Tl}$ sequences and hyperintensity in $\mathrm{T} 2$ sequences) in the GP, probably sparing the innermost part of the GPi (fig 1), but the rest of the brain appeared completely normal.

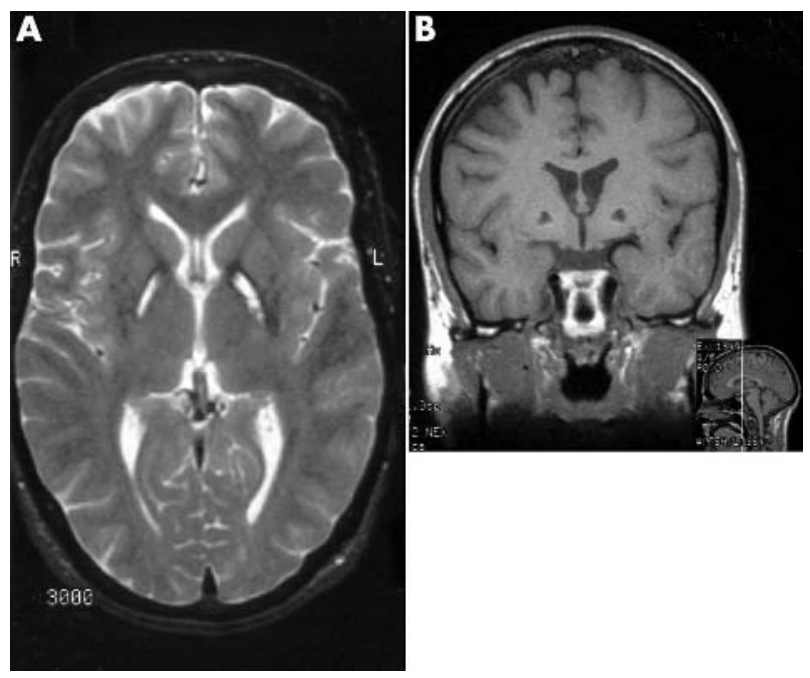

Figure 1 (A) T2 weighted axial MRI image showing hyperintense bilateral and symmetrical signal changes in the GP, probably sparing the innermost part of the GPi. (B) The same lesions are hypointense on T1 weighted coronal MRI image. The rest of the brain is normal on both sequences.

\section{METHODS}

To investigate different aspects of motor performance in our patient, and to compare him to healthy control subjects and patients with $\mathrm{PD}$, we deliberately chose tasks for which we already had data from these two groups. All the investigations reported below were performed three and a half years after the incident which gave rise to the bilateral GP lesions. The patient was 29 years old at the time of the investigations.

\section{Reaction time tasks}

Simple reaction time (SRT), uncued choice reaction time (CRT), and precued choice reaction time (pCRT) tasks were performed as previously described. ${ }^{3}$ The tasks were carried out first with the right and then the left hand. For each task, a block of 100 trials was completed with each hand. For each of the SRT, uncued CRT, and precued CRT tasks, 20 trials were given for each of the five S1-S2 intervals $(0,200,800$, 1600 , and $3200 \mathrm{~ms}$ ) in pseudorandom order, with an equal number of responses to each of the four response keys. Median IT was calculated for both hands and for each of the five S1-S2 intervals.

\section{Pegboard and finger tapping}

The pegboard and finger tapping tasks were performed as previously described. ${ }^{14}$ The pegboard task involved peg placement using the Purdue pegboard. ${ }^{15}$ The patient performed the task with the right and the left hand separately, and with both hands simultaneously. On each occasion, the patient had to place as many pegs as possible in a 30 second period. In the finger tapping task, the patient was required to tap a response button repetitively using the index finger for 30 second. The tapping task was performed with the right hand, the left hand, and bimanually. In addition to performing the tapping and pegboard tasks individually, the patient also performed these tasks concurrently, tapping with one hand while placing pegs with the other.

\section{Flex and squeeze tasks}

Flex and squeeze tasks were performed as previously described. ${ }^{16}{ }^{17}$ The patient sat with his forearm flexed at $90^{\circ}$, resting on a manipulandum, with his hand holding a vertical grip containing a strain gauge. The patient performed two types of movement, a $15^{\circ}$ flexion of the elbow and an isometric 30 Newton $(\mathrm{N})$ hand squeeze. Three conditions were assessed: simple flexion performed alone, the two movements performed simultaneously, and finally the two movements performed sequentially in the order squeeze then flex. For the sequential task, the inter-onset latency (IOL) between the onsets of the squeeze and flex was also determined.

\section{Movement related cortical potentials}

Movement related cortical potentials (MRCPs) were recorded as previously described. ${ }^{5}$ The patient was required to perform self-initiated joystick movements at the rate of once every 510 seconds, in a randomly chosen direction-left, right, forwards, or backwards. Twelve EEG channels were recorded. Electrodes were placed in three sagittal rows, according to the 10-20 international system and with three additional electrodes in FC sites (F3, FC3, C3, P3 on the left side, Fz, $\mathrm{FCz}, \mathrm{Cz}, \mathrm{Pz}$ on the midline, and F4, FC4, C4, P4 on the right side). EMG activity of the first dorsal interrosseous and the abductor pollicis brevis was recorded with surface electrodes. EEG activity was back-averaged off line after realignment of the traces to movement onset, a grand average being calculated for each hand. The early (NS1) and the late (NS2) components of the averaged MRCP were identified by visual inspection. The onset of the early component was set at the onset of the rise of the slope from baseline and the end at 
the point of change of the slope, which corresponded to the onset of the late component. The end of the late component corresponded to the time of onset of EMG activity; the slopes of each component were measured.

\section{Contingent negative variation}

Contingent negative variation (CNV) was recorded as above, only the task performed by the subject was different. This was a ready-go task in which a pair of tones was presented via headphones. The warning tone (S1) of $500 \mathrm{~Hz}$ was followed by an imperative stimulus ( $\mathrm{S} 2$, a tone of $1 \mathrm{kHz}$ ); the S1-S2 interval was 2.5 seconds. The subject was instructed to perform the left wrist extension task on hearing S2. The mean amplitude of the CNV from 500-1000 ms after S1 was measured.

\section{Cognitive function}

Our patient had neuropsychological assessment three years after his first admission to hospital. A battery of tests of cognitive function was used to assess IQ, memory, language, and visuospatial abilities and executive function. Current IQ was measured on the Wechsler Adult Intelligence ScaleRevised (WAIS-R). ${ }^{18}$ Estimates of premorbid IQ were obtained from the National Adult Reading Test (NART). ${ }^{19}$ Memory for words and faces was assessed with the Recognition Memory for Words (RMW) and Faces (RMF). ${ }^{20}$ To assess language and visuospatial abilities, the Graded Naming Test ${ }^{21}$ and the Silhouettes subtests of the Visual Object Space Perception ${ }^{22}$ battery were completed.

\section{RESULTS}

To allow comparison with the previously published data (in healthy controls and PD), all the results for the different reaction time tasks, and for the pegboard and finger tapping tasks, are given as mean values for the right and the left hand.

\section{Reaction time tasks \\ Unwarned and warned SRT tasks \\ Initiation times}

The mean (standard deviation) initiation time (IT) for the unwarned SRT was 456 (73) ms. The patient benefited from warning signals preceding the go-signal (at different intervals of $200-3200 \mathrm{~ms}$ ), and his ITs were relatively constantly around $350 \mathrm{~ms}$ (mean 344 (11) ms) after different warning signal intervals.

\section{Movement times}

The mean (SD) movement time (MT) for the unwarned SRT was 193 (SD 56) ms. When the warning signal preceded the go signal (at different intervals of 200-3200 ms), the mean (SD) MT was 188 (26) ms-that is, at about the same level as in the unwarned trials.

\section{Use of advance information \\ Unwarned SRT versus unwarned and uncued CRT}

The ability to prepare movements in advance was assessed by comparing ITs in the unwarned SRT and unwarned and uncued CRT trials. Because the nature of the response is known in advance of stimulus presentation in SRT it can be preprogrammed, which gives SRT a speed advantage over CRT. In the present case, the difference between the mean ITs in the unwarned and uncued CRT (mean 587 (SD 128) ms) and the unwarned SRT (mean 456 (SD 73) ms) was $131 \mathrm{~ms}$ (fig 2A).

\section{Precued CRT versus uncued CRT}

In a precued CRT task, a precue presented 200,800, 1600, or $3200 \mathrm{~ms}$ before a go signal provides full advance information
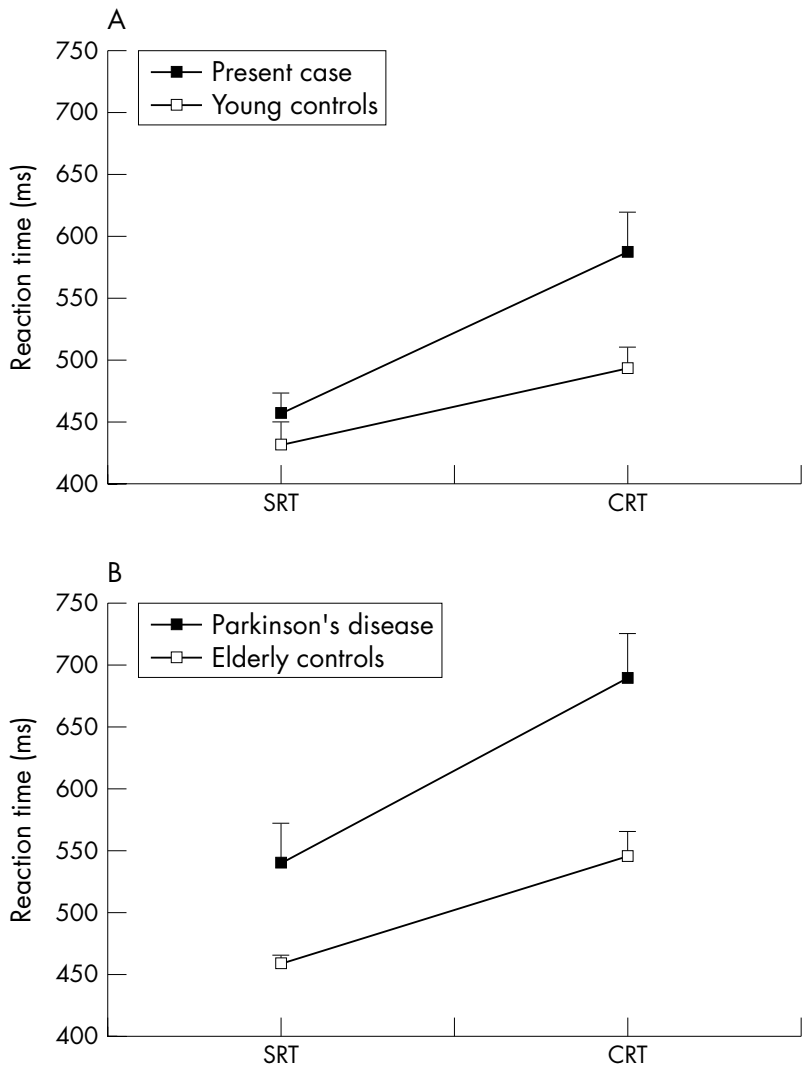

Figure 2 Reaction times (in milliseconds) in SRT and unwarned and uncued CRT paradigms. The present case and patients with PD have differentially greater slowness of CRTs ( $v$ SRTs) compared with their respective age matched controls. (A) Present case versus young controls. (B) Patients with PD versus elderly controls. The data for young controls is from Fuller and Jahanshahi, ${ }^{34}$ and the data for PD and elderly controls from Jahanshahi et al. ${ }^{3}$ Values represent means (SD). SRT, simple reaction time; CRT, choice reaction time (for more detailed explanation, see Methods and Results).

about the particular response required on that trial. If this advance information provided by the precue is used by the subject to pre-programme responses before the go signal, then precued CRTs should be faster than uncued and warned CRTs. On average, at different precue intervals, there was no difference between the mean ITs in the precued CRT (mean 489 (SD 93) $\mathrm{ms}$ ) and the uncued and warned CRT (mean 494 (SD 27)). Only for the $1600 \mathrm{~ms}$ interval was the precued CRT (mean 373 (SD 68)) clearly faster than the uncued CRT (mean 481 (SD 110)). Figure 3A summarises the reaction times in warned SRT, warned and uncued CRT, and precued CRT tasks. In terms of speed of movement execution during the various RT conditions, our case obtained mean MTs of $187.5 \mathrm{~ms}$ across the warned and unwarned SRT trials, a mean MT of $341.1 \mathrm{~ms}$ for the uncued CRT, and $186.1 \mathrm{~ms}$ for the precued CRT conditions.

\section{Performance on the pegboard and tapping tasks}

All the values given are means for the right and left hand. During the period of 30 seconds, the patient performed 185 unimanual taps. Bimanually, he was able to produce about the same number of taps (177 taps, 96\% of unimanual condition) compared with the unimanual conditions. The number of pegs inserted unimanually was 13 during the period of 30 seconds. Compared with unimanual performance, the number of pegs inserted decreased slightly during the bimanual condition ( 11 pegs, $85 \%$ of unimanual condition). 

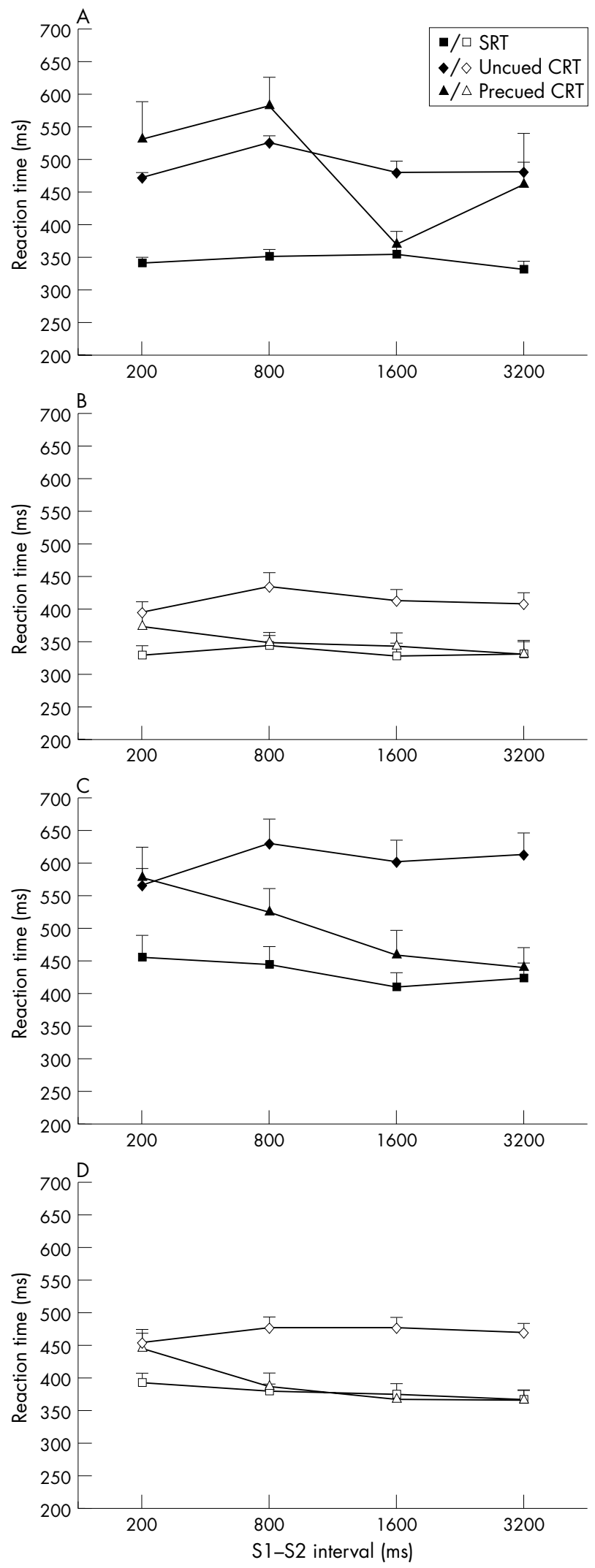

Figure 3 Reaction times (in milliseconds) in SRT, uncued CRT and precued CRT paradigms after different precue intervals (S1-S2 intervals). (A) Present case. (B) Young controls. (C) Patients with PD. (D) Elderly controls. The data for young and elderly controls are from Jahanshahi et $a^{\beta}$ and Fuller and Jahanshahi, ${ }^{34}$ respectively. The data for PD is from Jahanshahi et al. ${ }^{3}$ Values represent means (SD).
In a more complex task, in which the patient placed pegs with one hand, and did tapping with the other, the number of taps clearly decreased (104 taps, 56\% of unimanual condition). However, the number of pegs inserted remained at the same level compared with the unimanual condition (12 pegs, 92\% of unimanual condition). The results of the pegboard and finger tapping tasks are summarised in table 1.

\section{Performance of the flex and squeeze tasks}

Movement times for the two tasks performed separately are for the right hand. In the simultaneous and sequential tasks, hand squeeze was performed with the left hand, and elbow flexion with the right hand. In the sequential task, hand squeeze was performed first, followed by elbow flexion. The movement time for squeeze alone was $158 \mathrm{~ms}$. Hand squeeze performance slowed down to some extent when performed simultaneously with elbow flexion (190 ms; $20 \%$ increase in movement time). The movement time for elbow flexion alone was $209 \mathrm{~ms}$. Elbow flexion performance remained at about the same level when performed simultaneously with hand squeeze (226 ms; $8 \%$ increase in movement time). In the sequential task, performance of elbow flexion clearly slowed down ( $297 \mathrm{~ms} ; 42 \%$ increase in movement time). The results of the flex and squeeze tasks are summarised in table 2 .

\section{Movement related cortical potentials}

Compared with the previous data for healthy controls, ${ }^{5}$ the movement related cortical potentials (MRCPs) preceding voluntary joystick movements were abnormal. The first component (NS1) (usually starting at about 1.5 seconds before movement onset) was absent prior to movement of either hand, as shown by the flat early slope. The later (NS2) component was present (amplitude of $5 \mathrm{uV}$ on the left; amplitude of $7 \mathrm{uV}$ on the right) (fig 4 ).

\section{Contingent negative variation}

There was no recordable contingent negative variation (CNV) in an S1/S2 task using an interval of 2.5 seconds and an imperative movement of wrist extension on the left (fig 5).

\section{Cognitive function}

Three years after the insult, the Verbal and Performance IQs of our case were 102 and 106, respectively. Both of these were in the average range. On the NART, his estimated premorbid IQ of 117 was in the high average range. Comparison of the verbal IQ on the WAIS-R and the premorbid estimate of IQ from the NART suggested moderate cognitive under

Table 1 Performance of pegboard and finger tapping tasks under unimanual, bimanual, and dual task conditions

\begin{tabular}{|c|c|c|c|}
\hline & Present case & Controls $(n=13)$ & $P D(n=7)$ \\
\hline Age (years) & 29 & 38 (range 21-62) & $61(10)$ \\
\hline Pegboard (unimanual) & 13 & 16 (2) & $9(2)$ \\
\hline Pegboard (bimanual) & 11 & $14(2)$ & $7(3)$ \\
\hline Unimanual pegboard (\%) & 85 & $87(8)$ & $71(19)$ \\
\hline Tapping (unimanual) & 185 & $173(19)$ & $113(22)$ \\
\hline Tapping (bimanual) & 177 & $165(26)$ & $108(30)$ \\
\hline Unimanual tapping (\%) & 96 & $94(10)$ & 95 (13) \\
\hline Pegboard (with tapping) & 12 & $15(2)$ & $10(2)$ \\
\hline Unimanual pegboard (\%) & ) 92 & $93(9)$ & $106(9)$ \\
\hline Tapping (with pegboard) & ) 104 & $150(22)$ & $61(31)$ \\
\hline Unimanual tapping (\%) & 56 & $87(10)$ & $55(27)$ \\
\hline
\end{tabular}

PD data is taken from Brown et al, ${ }^{14}$ and data for control subjects from Fuller and Jahanshahi. ${ }^{77}$ Data shown is the mean of the right and left hand. PD and control data is expressed as mean (SD). 
Table 2 Movement times (in milliseconds) in flex and squeeze tasks

\begin{tabular}{|c|c|c|c|}
\hline & Present case & Controls $(n=9)$ & $P D(n=10)$ \\
\hline Age (years) & 29 & $47(16)$ & $58(10)$ \\
\hline Squeeze (alone) & 158 & $156(23)$ & $221(29)$ \\
\hline Squeeze (simultaneous) & 190 & $156(27)$ & 309 (77) \\
\hline Increase in time (\%) & 20 & 0 & 40 \\
\hline Squeeze (sequential) & 182 & $150(25)$ & $254(38)$ \\
\hline Increase in time (\%) & 4 & -4 & 15 \\
\hline Flexion (alone) & 209 & $229(41)$ & $349(77)$ \\
\hline Flexion (simultaneous) & 226 & $216(30)$ & 557 (154) \\
\hline Increase in time (\%) & 8 & -6 & 60 \\
\hline Flexion (sequential) & 297 & $244(35)$ & $445(110)$ \\
\hline Increase in time (\%) & 42 & 7 & 28 \\
\hline $\mathrm{IOL}$ & 212 & $244(33)$ & $425(46)$ \\
\hline
\end{tabular}

Data for separate (alone) tasks are for the right hand. In the simultaneous and sequential tasks, hand squeeze was performed with the left hand, and elbow flexion with the right hand. In the sequential task, hand squeeze was performed first, followed by elbow flexion. Percentage increase in time values refer to the respective alone values. PD and control data are taken from Benecke et $\mathrm{al}^{77}$ and expressed as mean (SD).

IOL, interonset latency in the sequential task.

functioning. His memory for words (RMW 41/50 correct, 10th percentile) and faces (RMF $37 / 50$ correct 5 th percentile) were impaired. On the Graded Naming Test he obtained a score $(22 / 30)$ in the superior range. His visuospatial abilities (VOSP Silhouettes score 25/30) were in the average range. On a relatively easy test of set shifting, the Weigl test, he performed adequately and correctly sorted by both rules.

\section{DISCUSSION \\ Clinical characteristics of parkinsonism caused by lesions of globus pallidus}

Bilateral isolated lesions of globus pallidus (GP) are rare. In a large series of basal ganglia lesions reported by Bhatia and Marsden, ${ }^{11}$ there were only 15 out of 240 such cases. Only four of these 15 cases $(27 \%)$ had parkinsonism. One presented with parkinsonism alone, ${ }^{23}$ whereas others had additional abulia ${ }^{24}$ or abulia associated with either speech disorder or obsessive-compulsive behaviour. ${ }^{25}$ In addition to these, there are only a few other cases reported with parkinsonism caused by bilateral pallidal lesions. Hawker and Lang $^{26}$ reported a case with severe akinetic rigid syndrome after sudden respiratory arrest and subsequent bilateral pallidal lesions. Fève et $a 2^{27}$ and Bucher et $a l^{28}$ reported four cases each, and Haaxma et al ${ }^{12}$ described one case, who had an additional lesion of the right substantia nigra. After the initial insult, the symptoms of parkinsonism of our case developed after a delay and subsequently progressed. We believe, however, that this was due to his pallidal lesions as the development of delayed onset cases of parkinsonism or other extrapyramidal symptoms after acute insults of varying causes have been well documented before. ${ }^{29-31}$ It is also unlikely that the parkinsonism was due to alcoholism as it has been reported that alcohol withdrawal induced parkinsonism is likely to disappear during relatively short follow up, ${ }^{32}$ which was not the case with our patient.

As can be seen from table 3, the most common causes for these lesions were either brain hypoxia or carbon monoxide (CO) poisoning. Regarding the nature of parkinsonism, it appears that, in these published cases, axial motor problems (gait and speech disorder with possible freezing and postural instability) seemed to dominate over brady/akinesia (defined mainly by rapid alternating (distal) limb movements), rigidity, and tremor of the limbs (table 3 ). This was also the case with our patient, who had a speech problem, "fast micrographia", ${ }^{13}$ and impaired postural reflexes, but no distal limb akinesia or tremor. However, the clinical picture of

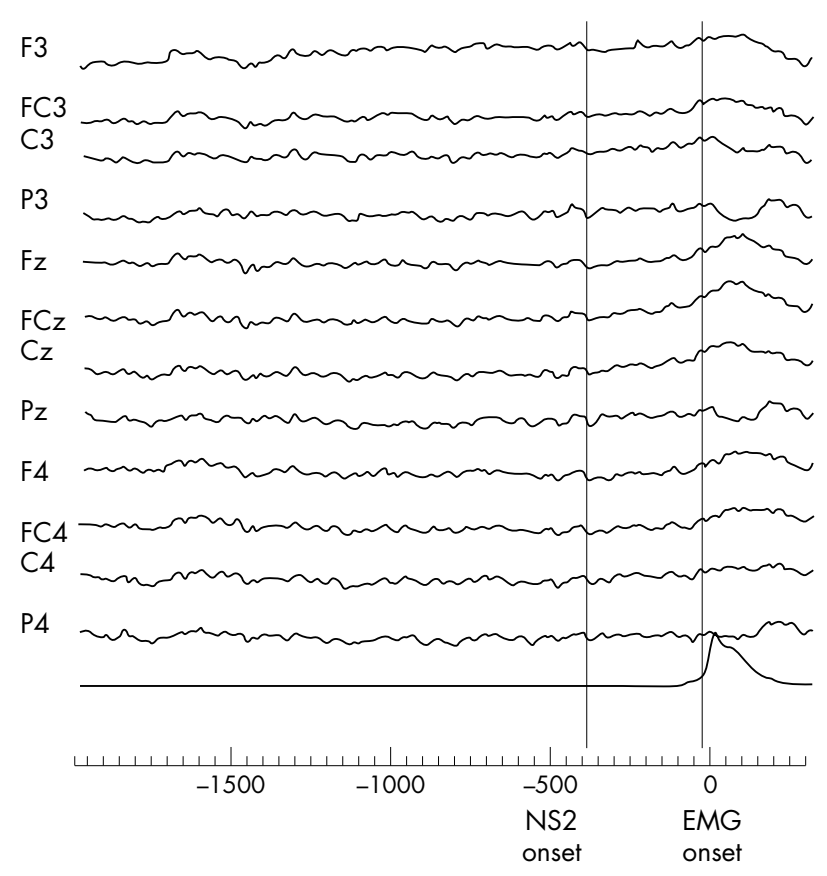

Figure 4 Data for movement related cortical potential (MRCP) before self paced voluntary movement.

parkinsonism of our case differed from that of typical PD, as the main clinical findings in $\mathrm{PD}$ are rest tremor, distal bradykinesia, and limb rigidity. The case reported by Haaxma et $a l^{12}$ who also underwent detailed investigations of motor function, presented with a striking gait disorder, postural instability, and general slowness. It is also notable that most of these cases had lesions affecting both the internal (GPi) and external (GPe) segments of the globus pallidus, or alternatively, the lesions were restricted to GPe only. It has been suggested that, within GP, it is mainly the lesions of the

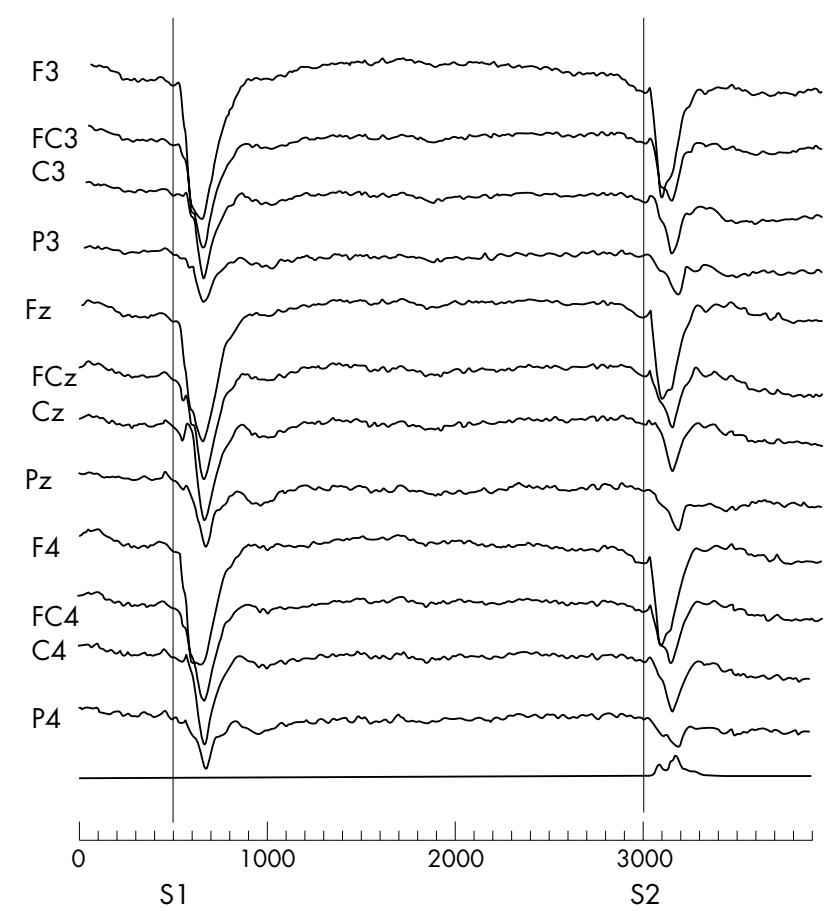

Figure 5 Contingent negative variation. 


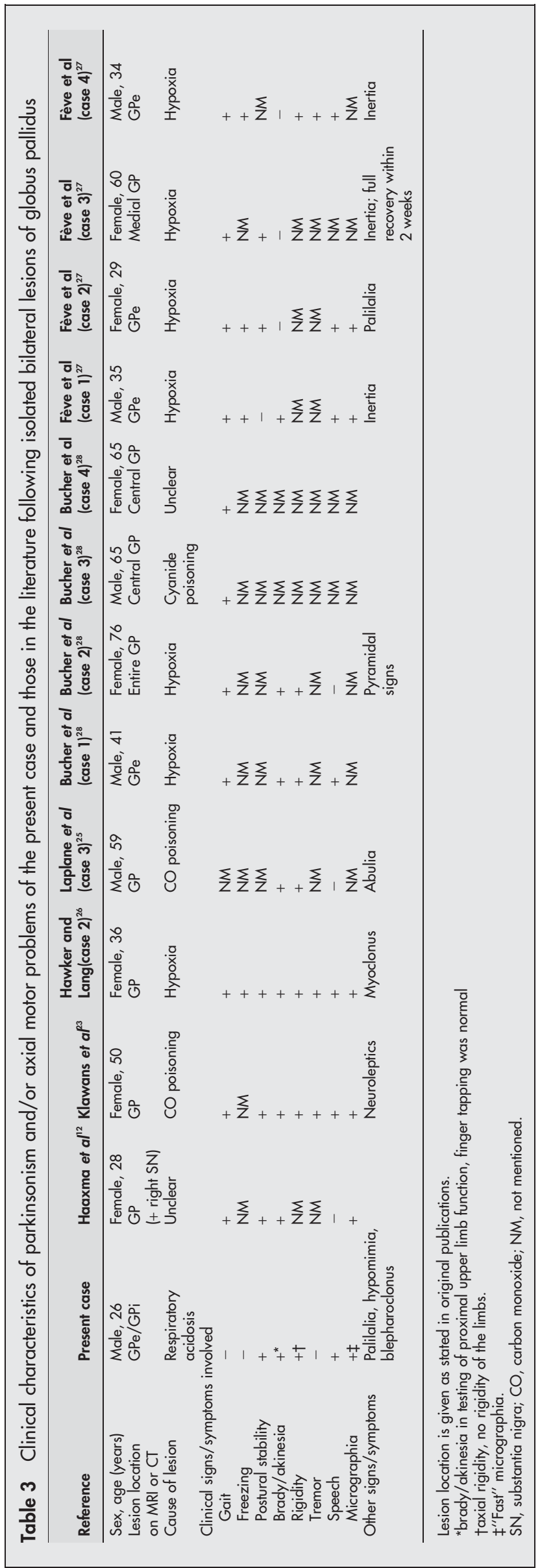

external part that are responsible for parkinsonism. ${ }^{28}$ In contrast, lesions restricted to GPi may predominantly give rise to dystonia. ${ }^{28} 33$

\section{Motor performance examined with different experimental tasks}

The motor performance of the present case is compared, when available, to the previously published data of normal control subjects and patients with PD.

\section{Preparation for movement}

There is general consensus that SRTs are prolonged in PD. ${ }^{7}$ The present subject had slightly slower reaction times (ITs) in unwarned (mean 456 (SD 73) ms) and warned (mean 344 (11) ms) SRT trials compared to the control group of the same age range (mean age 39 (SD 10) years), who had mean unwarned SRTs of about $430 \mathrm{~ms}^{34}$ Although his SRTs were slightly faster compared with patients with PD (mean age 64 (SD 8) years), who had mean warned SRTs of about $400 \mathrm{~ms}$, the relative slowness of SRTs in our case compared with age matched control subjects was in the same range of magnitude (about $50 \mathrm{~ms}$ ) to that reported for patients with PD compared with their age matched control subjects. ${ }^{3}$ This suggests that the unwarned SRTs of our young patient with bilateral lesions of GP were prolonged in a similar fashion as previously reported in PD. ${ }^{1-3}$ The same was true for unwarned and uncued CRTs, which showed differentially greater slowness than SRTs (fig 2.) in the present case relative to the age matched controls (difference of $94 \mathrm{~ms}$ for CRTs compared with difference of $15 \mathrm{~ms}$ for SRTs between our case and young controls) and in PD patients compared with their elderly age matched controls (difference of $145 \mathrm{~ms}$ for CRTs compared with $81 \mathrm{~ms}$ for SRTs between PD and elderly controls). ${ }^{34}$ Although the methods to study simple and choice reaction times used by Haaxma et al ${ }^{12}$ were somewhat different from ours, their overall results were in line with our case, as both the simple and choice reaction times of their case were slower compared with control subjects.

In healthy controls, SRTs are faster than $\mathrm{CRTs}^{34}$ in part because, due to its invariant nature, in SRT subjects have the possibility to prepare in advance the movement they will have to perform at the time of the go-signal. Our case also benefited from this advance information, as his SRTs were clearly faster than his CRTs (fig 2). This has also been reported in PD. ${ }^{1-3}$ The magnitude of the CRT-SRT difference in our case ( $130 \mathrm{~ms}$ ) was similar to that previously reported in PD (a mean difference of 148 (SD 71) ms), and clearly greater than that reported in controls of the same age range (mean 60 (SD 5l) ms), or in controls age matched with PD (mean 86 (SD 70) ms). ${ }^{34}$ As evident from figure 2 and as noted above, the greater CRT-SRT difference in our case relative to young controls and in PD patients relative to elderly controls is due to the fact that their CRTs show a differentially greater slowness than their SRTs compared with age matched controls. Thus, the current case with bilateral pallidal lesions also shows the differential slowing of CRTs previously found ${ }^{3}$ and replicated by $\mathrm{us}^{35}$ in patients with Parkinson's disease.

Fully cued CRTs provide another way to study the subject's ability to prepare movements in advance-in particular the time needed to use advance information about the forthcoming movement to speed up response initiation. Healthy control subjects can take advantage of the precue to speed up their CRTs to the level of SRTs, if this information is presented at least $800 \mathrm{~ms}$ before the go signal ${ }^{34}$ (fig 3B). Although PD patients can also benefit from a precue, they seem to be slower in preparing expected precued responses, since they require a longer S1-S2 interval of $3200 \mathrm{~ms}$ to do this $^{3}$ (fig 3C). At only one interval, $1600 \mathrm{~ms}$, was the precued 
CRT of our case faster than his warned and uncued CRT, and equivalent to his SRT, but he failed to maintain this benefit with a longer interval of $3200 \mathrm{~ms}$ (fig 3A). This suggests that, as with parkinsonian patients (but in contrast to control subjects), our case did not benefit from the extra information about the hand and direction of movement provided by the precue.

Preparation to move can also be investigated by recording the activation of specific brain areas before self paced voluntary movements. Movement related cortical potentials (MRCP) have two major components. The early component (early BP or NS1) appears 1-2 seconds before movement, and the second component (late BP or NS2) begins about $650 \mathrm{~ms}$ before the onset of EMG activity, and rises more steeply than the earlier one. It has been shown that the first component is reduced in patients with PD when assessed off medication..$^{4-6}$ As in PD, the MRCPs of our case were abnormal as the first component was absent prior to the movement of either hand; the later component was present. The CNV is a slow negative potential occurring between a warning (S1) and an imperative (S2) stimulus, and it reflects processes related to preparation of a forthcoming movement and anticipation of the imperative stimulus. In PD, the amplitude of the CNV is reduced and correlates with the severity of the disease. ${ }^{7}$ Our case had no recordable CNV when an S1-S2 interval of 2.5 seconds was used.

\section{Execution of simple and complex movements}

In the RT tasks, the movement times (MTs) provide information about the speed of execution of simple aimed movements. In general, the MTs in SRT tasks are faster than in CRT tasks both in healthy controls and PD, but MTs do not differ significantly between precued and uncued CRT conditions. ${ }^{34}$ Compared with age matched controls, patients with PD have slower MTs. ${ }^{3}$ On average, in the SRT task, the MTs of the present case were similar to those of young age matched controls. ${ }^{34}$ In contrast, his MTs in the uncued CRT task were even slower than those of the elderly control subjects. ${ }^{3}$ In fact our case showed an abnormal pattern of MTs, in that his MTs for the precued CRT task (mean $186.1 \mathrm{~ms}$ ) were considerably faster (difference of $155 \mathrm{~ms}$ ) than his MTs for the uncued CRT condition (mean 341.1) and similar to his MTs for the SRT task (mean $187.5 \mathrm{~ms}$ ). This pattern was not previously seen in either of the young or old control groups or the patients with PD. ${ }^{34}$

Whereas the RT tasks provided fixed start and end points for the movement execution, the movements in the elbow flexion and hand squeeze tasks were self-terminated. Our case was slower in executing aimed movements with a fixed end point as his MTs in the SRT and uncued CRT tasks were slow, although he performed individual elbow flexion and hand squeeze movements at a normal speed. This contrasts with previous findings in parkinsonian patients, in whom it has been suggested that they may have greater difficulties in executing self-terminating movements. ${ }^{36}$

For patients with PD, simple repetitive movements such as finger tapping and peg placement tasks (involving distal movements) and simultaneous or sequential movements (for example, flex and squeeze or concurrent peg placement and tapping) are more difficult to execute than simple and single movements. ${ }^{7}$ Furthermore, the more complex the task, the greater the deterioration in the performance of PD patients compared with control subjects, as indicated by a marked drop of the number of taps during simultaneous placing of pegs with the other hand. ${ }^{14}$ Indeed, this dual task demonstrated one of the most relevant similarity between PD patients and our case (table 1, bottom line). Similarly, as in $\mathrm{PD}^{37}$ the speed of elbow flexion in our case slowed down in the sequential task (table 2). Compared with PD, there were, however, some differences in simultaneous and sequential flex and squeeze tasks. In contrast to the previously published data in $\mathrm{PD}^{16}{ }^{17} 37$ the speed of elbow flexion in our case was faster (and about the same as in middle aged (mean age 47 years, range 31-67 years) control subjects), and it remained the same whether executed on its own or simultaneously with a squeeze of the other hand. In addition, the IOL between the sequential tasks was much shorter than in $\mathrm{PD}$, and slightly shorter than in middle aged control subjects, ${ }^{37}$ - that is, it appeared to be normal. In this regard, the performance of a similar case reported earlier ${ }^{12}$ resembled PD more closely, as the speed of elbow flexion in that case slowed down during both the simultaneous and sequential tasks, and the IOL was about three times longer compared with controls. The reasons for the different results between these two cases with bilateral lesions of GP are not known. One possible explanation could be that whereas our case had isolated GP lesions, the case reported by Haaxma et al ${ }^{12}$ had an additional lesion in the right substantia nigra. It is also possible that the size and the exact sites of the lesions within GP were different in these two cases.

\section{Cognitive function}

Some of the previous studies have suggested that bilateral pallidal leasons may give rise to deficits on tests of executive function and/or passivity, inertia and lack of initiative (abulia). ${ }^{12} 2527$ Abulia and apathy can also be seen in long standing PD. ${ }^{38}$ However, our patient had no clinical evidence of abulia. Our patient had normal language and visuospatial abilities, but the neuropsychological assessment indicated memory impairment. We believe that the cognitive findings of our case may be a result of the GP lesions, as memory disturbance and deficits of recognition memory in patients with pallidal lesions have been previously reported..$^{12}{ }^{27}$ However it is also possible that the memory deficits of our subject may have resulted from the alcohol abuse and possible changes in other brain areas not visible in the MRI.

\section{Comparison with the effects of pallidal surgery in PD}

In the light of the present results, it is also interesting to look at the effects of pallidal surgery (unilateral pallidotomy) on the same experimental measures of motor function, and the effects of surgery on the contralateral side are discussed below. Unilateral pallidotomy has been reported to improve (especially ITs) SRTs and CRTs, ${ }^{39}{ }^{40}$ although there is one study showing improvement of MTs, but not ITs, in these tasks. ${ }^{41}$ Interestingly, however, the IT in precued CRT improved after pallidotomy with a precue interval of $800 \mathrm{~ms}$, but not with shorter or longer intervals. ${ }^{41}$ This resembles the results of precued CRT of our case (see above). In addition, repetitive hand movements, such as pronation/ supination and finger tapping, and hand dexterity evaluated by pegboard test have been reported to be improved after pallidotomy. ${ }^{39} 40$ In the study by Limousin et al, ${ }^{41}$ pallidotomy had no significant effects on repetitive, distal, and fine movements measured by pegboard and finger tapping tasks, whereas it improved movement times in simple and simultaneous elbow flexion tasks. The use of different methods, the exact lesion sites within GP, and the proposed functional somatotopy of GP are likely to explain some of the discrepancies found in the above studies. ${ }^{43-45}$ However, in most of these studies, pallidotomy seemed to improve many of the experimental motor tasks that are abnormal in patients with PD and our case (compared with age matched controls). This gives indirect evidence that altered firing patterns of GP contribute to the execution of different motor tasks in PD. 


\section{What is the functional status of the lesioned GP in our previously healthy subject?}

A general assumption is that if a specific nucleus is lesioned, either deliberately or by some disease related event, it becomes functionally silent. However, this is perhaps not the case. Firstly, most of the different measures of motor performance in our case with bilateral GP lesions were similar to those reported previously in patients with PD. Secondly, although there is widespread pathology in the PD brain, and although the principal lesion in PD is the degeneration of the nigrostriatal pathway, it is the resultant abnormal pallidal output that is generally considered to be one of the most crucial factors contributing to the motor symptoms. ${ }^{8}{ }^{46}$ In $\mathrm{PD}$, in conjunction with being functionally overactive with increased firing rate, the current pathophysiological models suggest that it is perhaps the abnormal firing pattern of the GP that is even more important-that is, the pallidal output becomes "noisy". ${ }^{46}$ Given that the pallidal output is perhaps the only pathologically abnormal factor shared by both our subject and patients with $\mathrm{PD}$, and their similar performance on many tests of motor function, it is tempting to speculate that the functional status of the GP is similar in our case and in patients with PD. This suggests that, instead of being functionally silent, the pallidal output in our case with an acquired pallidal lesion may be "noisy", as in PD. This idea is supported by a recent report, in which patients with pallidal lesions (who had developed either parkinsonism or dystonia as a result of their lesions) were studied. Using functional MRI, Bucher et $a^{28}$ found that the pallida of these patients were still functionally active despite being lesioned, but direct electrophysiological recordings of these cases would be needed to confirm this. Taking into account the present results, the above findings on pallidal function, and the positive effects of pallidal surgery in PD, it seems possible that whereas the previously normal GP in a healthy person could be damaged and its output made "noisy" by a lesion, pallidotomy could normalise to some extent the "noisy" GP output in PD. It is also possible that if the GP is not entirely damaged (as is often the case), the nonlesioned part of the GP may become functionally unstable (noisy) and contribute to the abnormal output of the GP (together with the lesioned area). Finally, we compared the performance of our case with patients with $\mathrm{PD}$, as we had data from this group. However, these results do not have to be specific to patients with pallidal lesions or PD. Indeed, other extrapyramidal conditions with pallidal pathology could have similar results on the different tasks evaluated.

\section{Note added in proof:}

It was recently reported that in experimental conditions in rats, damage to globus pallidus can induce nigral dopaminergic cell death (Wright et al. Eur J Neurosci 2004;20:173744.

\section{ACKNOWLEDGEMENTS}

This study was supported by Maire Taposen Säätiö and Suomen Parkinson Säätiö.

\section{Authors' affiliations \\ M Kuoppamäki, Department of Neurology, Satakunta Central Hospital, and Satakunnan Neurologipalvelu Oy, Pori, Finland \\ J C Rothwell, N Quinn, K P Bhatia, M Jahanshahi, Sobell Department of Motor Neuroscience and Movement Disorders, Institute of Neurology, London, UK \\ R G Brown, Department of Psychology, Institute of Psychiatry, King's College London, London, UK}

Competing interests: none declared

\section{REFERENCES}

1 Stelmach GE, Worringham CJ, Strand EA. Movement preparation in Parkinson's disease: the use of advance information. Brain 1986; 109:1179-94.

2 Sheridan MR, Flowers KA. Movement variability and bradykinesia in Parkinson's disease. Brain 1990;113:1149-61.

3 Jahanshahi M, Brown RG, Marsden CD. Simple and choice reaction time and the use of advance information for motor preparation in Parkinson's disease. Brain 1992;115:539-64.

4 Dick JPR, Rothwell JC, Day BL, et al. The Bereitschaftpotential is abnormal in Parkinson's disease. Brain, 1989; 112, 233-44.

5 Touge T, Werhahn KJ, Rothwell JC, et al. Movement-related cortical potentials preceding repetitive and random-choice hand movements in Parkinson's disease. Ann Neurol 1995;37:791-9.

6 Jahanshahi M, Jenkins IH, Brown RG, et al. Self-initiated versus externally triggered movements. I. An investigation using measurement of regional cerebral blood flow with PET and movement-related potentials in normal and Parkinson's disease subjects. Brain 1995;118:913-33.

7 Berardelli A, Rothwell JC, Thompson PD, et al. Pathophysiology of bradykinesia in Parkinson's disease. Brain 2001;124:2131-46.

8 Obeso JA, Rodríguez-Oroz MC, Rodríguez M, et al. Pathophysiology of basal ganglia in Parkinson's disease. Trends Neurol Sci 2000;23(Suppl.):S8-S19.

9 Wichmann T, Smith Y, Vitek JL. Basal ganglia anatomy and physiology. In: Factor SA, Weiner WJ, eds. Parkinson's disease.Diagnosis and clinical management. New York: Demos, 2002:211-31.

10 Barron KD. Pathology. In: Factor SA, Weiner WJ, eds. Parkinson's disease. Diagnosis and clinical management. New York: Demos, 2002:183-93.

11 Bhatia KP, Marsden CD. The behavioural and motor consequences of focal lesions of the basal ganglia in man. Brain 1994;117:859-76.

12 Haaxma R, van Boxtel A, Brouwer WH, et al. Motor function in a patient with bilateral lesions of the globus pallidus. Mov Disord 1995;10:761-77.

13 Quinn N, Kuoppamäki M. Fast micrographia and pallidal pathology. Mov Disord 2003; 18:1067-9.

14 Brown RG, Jahanshahi M, Marsden CD. The execution of bimanual movements in patients with Parkinson's, Huntington's and cerebellar disease. J Neurol Neurosurg Psychiatry 1993;56:295-7.

15 Purdue Research Foundation. Purdue pegboard test: examination manual. Chicago: Science Research Associates, 1948.

16 Brown RG, Limousin-Dowsey P, Brown P, et al. Impact of deep brain stimulation on upper limb akinesia in Parkinson's disease. Ann Neurol 1999:45:473-88

17 Benecke R, Rothwell JC, Dick JPR, et al. Performance of simultaneous movements in patients with Parkinson's disease. Brain 1986;109:739-57.

18 Wechsler D. Wechsler Adult Intelligence Scale - Revised. Manual. New York: The Psychological Corporation, 1981.

19 Nelson HE. National adult reading Test (NART). Manual, Second Edition. NFER-NELSON, 1992

20 Warrington EK. Recognition Memory for Faces and Words. Manual. NFERNELSON, 1984.

21 McKenna P, Warrington EK. Graded naming test. Manual. NFER-NELSON, 1983.

22 Warrington EK, James M. Visual Object and Space Perception Battery (VOSP). Thames Valley Test Company, 1991.

23 Klawans HL, Stein RW, Tanner CM, et al. A pure parkinsonian syndrome following acute carbon monoxide intoxication. Arch Neurol 1982;39:302-4.

24 Grinker RR. Parkinsonism following carbon monoxide poisoning. J Nerv Ment Dis 1926:64:18-28.

25 Laplane D, Levasseur M, Pillon B, et al. Obsessive-compulsive and other behavioral changes with bilateral basal ganglia lesions. Brain 1989;112:699-725.

26 Hawker K, Lang AE. Hypoxic-ischemic damage of the basal ganglia. Case reports and a review of the literature. Mov Disord 1990;5:219-24.

27 Fève AP, Févelon $G$, Wallays $C$, et al. Axial motor disturbances after hypoxic lesions of the globus pallidus. Mov Disord 1993;8:321-6

28 Bucher SF, Seelos KC, Dodel RC, et al. Pallidal lesions. Structural and functional magnetic resonance imaging. Arch Neurol 1996;53:682-6.

29 Ginsberg MD. Delayed neurological deterioration following hypoxia. Adv Neurol 1979;26:21-44.

30 Bhatt MH, Obeso JA, Marsden CD. Time course of postanoxic akinetic-rigid and dystonic syndromes. Neurology 1993;43:314-17.

31 Kuoppamäki M, Bhatia KP, Quinn N. Progressive delayed-onset dystonia after cerebral anoxic insult in adults. Mov Disord 2002;17:1345-9.

32 Shandling M, Carlen PL, Lang AE. Parkinsonism in alcohol withdrawal: a follow-up study. Mov Disord 1990;5:36-9.

33 Münchau A, Mathen D, Cox T, et al. Unilateral lesions of the globus pallidus: report of four patients presenting with focal or segmental dystonia. $J$ Neurol Neurosurg Psychiatry 2000;69:494-8.

34 Fuller R, Jahanshahi M. Impairment of willed actions and use of advance information for movement preparation in schizophrenia. J Neurol Neurosurg Psychiatry 1999;66:502-9.

35 Brown RG, Jahanshahi M, Marsden CD. Response choice in Parkinson's disease. The effects of uncertainty and stimulus-response compatibility. Brain 1993; 116:869-85.

36 Flowers KA. Visual 'closed-loop' and 'open-loop' characteristics of voluntary movement in patients with parkinsonism and intention tremor. Brain 1976;99:269-310

37 Benecke R, Rothwell JC, Dick JPR, et al. Disturbance of sequential movements in patients with Parkinson's disease. Brain 1987; 110:361-79. 
38 Starkstein SE, Mayberg HS, Preziosi TJ, et al. Reliability, validity, and clinical correlates of apathy in Parkinson's disease. J Neuropsychiatry Clin Neurosci 1992;4:134-9

39 Hayashi $\mathbf{R}$, Hashimoto $T$, Tada $T$, et al. Effects of unilateral pallidotomy on voluntary movement, and simple and choice reaction times in Parkinson's disease. Mov Disord 2003;18:515-23.

40 Jankovic J, Ben-Arie L, Schwartz K, et al. Movement and reaction times and fine coordination tasks following pallidotomy. Mov Disord 1999;14:57-62.

41 Limousin P, Brown RG, Jahanshahi $M$, et al. The effects of posteroventral pallidotomy on the preparation and execution of voluntary hand and arm movements in Parkinson's disease. Brain 1999;122:315-27.

42 Levy $R$, Lang $A E$, Hutchison WD, et al. Simultaneous repetitive movements following pallidotomy or subthalamic deep brain stimulation in patients with Parkinson's disease. Exp Brain Res 2002;147:322-31.
43 Krack P, Pollak P, Limousin P, et al. Opposite motor effects of pallidal stimulation in Parkinson's disease. Ann Neurol 1998;43:180-92

44 Gross RE, Lombardi WJ, Lang AE, et al. Relationship of lesion location to clinical outcome following microelectrode-guided pallidotomy for Parkinson's disease. Brain 1999;122:405-16.

45 Kishore A, Panikar D, Balakrishnan S, et al. Evidence of functional somatotopy in GPi from results of pallidotomy. Brain 2000;123:2491-500

46 Wichmann T, DeLong MR. Functional and pathophysiological models of the basal ganglia. Curr Opin Neurobiol 1996;6:751-8.

47 Fuller R, Jahanshahi M. Concurrent performance of motor tasks and processing capacity in patients with schizophrenia. J Neurol Neurosurg Psychiatry 1999;66:668-71.

\section{Register now!}

10th European Forum on Quality Improvement in Health Care

13-15 April 2005, ExCel Conference Centre, London

For further information on how to register please go to:

http://www.quality.bmipg.com 